discharge-will be about 0.62 . When, however, the opening is approached by a funnel-shaped passage the coefficient is greatly increased. The exact increase depends upon the inclination of the walls of the funnel: thus, when the inclination is from $48^{\circ}$ to $50^{\circ}$, the coefficient is 0.847 ; when from $13^{\circ}$ to $17^{\circ}$ it is 0993 . What the coefficient is in funnel-shaped passages with chink-like orifices of exit has not, so far as I know, been ascertained, but I believe it will be fourd to differ very little from that of a regular cone with a circular orifice of exit; and if such is the case it must be assumed that the funnel-shaped openings met with in stenosis of the mitral and aortic valves tend to facilitate the flow of blood through them.

Next as to the flow of fluid through chinks in flat diaphragms. It has been seen that when the orifice is perfectly circular in such a partition, the coefficient is small. One would accordingly not expect to find a cardiac orifice occluded by a membranous partition perforated in its centre by a circular orifice. Such an orifice I have neither seen nor heard of. When a membranous diaphragm stretches across a cardiac orifice the opening in it always, so far as I know, takes the form of a slit, and in searching for the coefficient of such orifices I was accordingly not surprised to find that I had anticipated rightly - that it is "much greater for long, narrow orifices than for circular."'

From the above considerations it is manifest that the funnelshaped and slit-like orifices tend to minimise the evils of stenosis. In them there is seen an endeavour, as it were, to make the best of a bad state of affairs. Having explained the advantage attaching to them, the mode of $t$ ' eir production may be examined. I believe the proposition that "a fluid flowing more or less continuously through an aperture in a plastic membrane tends to mould that aperture and that membrane so as to give the largest available coefficient of discharge" is deducible from physical laws. Apart from this, however, it will be observed that the agglutination of the adjacent edges of the mitral valves tends to produce a funnel the apex of which projects in the direction of the current, and the same remarks apply to the aortic valves. Hence from the very beginning of the stenosis there is often a tendency to the funnelshaped opening; but this tendency of the agglutinated valves to form a funnel is by no means an essential factor in its production. As the mutilated valves contract and close up the valvular orifice they tend to form a membranous diaphragm across it. Now, a plastic membrane with a small opening in its centre naturally tends to project in the form of a funnel if one side is subjected to fluid pressure. Anyone may confirm this a priori deduction by tightly stretching a thin piece of gutta-percha over the nozzle of a tap, piercing an orifice in its centre and then turning on the water. In a similar way the blood flowing against the stenosed mitral and aortic valves tends to protrude them cone-wise in the direction of the current. Why, then, is not the cone always observed in stenosis? In order to explain this it must be borne in mind that the form of the stenosed orifice depends upon the action and reaction of two sets of forces. On the one hand, there are the vital activities of the tissue surrounding the diseased orifice tending to mould it into a certain shape ; and, on the other hand, the pressure of the blood passing through the constricted orifice tending to shape it in such wise as to secure the largest available discharge. When the former forces are so powerful as to lead to the formation of a more or less flat partition stretching across the original orifice, as in extreme mutilation of the mitral valves going on to excessive cicatricial constriction, or abundant atheromatous deposit ("ossification") in the aortic cusps, then the orifice takes the form of a shit or chink, which allows more fluid to pass through than a circular opening of the same area.

It will thus be seen that the laws regulating the flow of fluid through orifices have important bearings upon the pathology of the circulation. I have here only touched upon a few of these bearings in order that the issues may be kept clear. Devonshire-street, Portland.place, $\mathbf{w}$.

${ }^{1}$ See Mr. Hamilton Smith's work on the Flow of Water through Pipes, Orifices, \&c., p. 43.

The Pasteur Institute in Bengal.-A Reuter's telegram from Calcutta states that the Bengal branch of the Pasteur Institute was successfully inaugurated on Jan. 30th in the presence of a large company. After the ceremony subscriptions to a considerable amount were promised.

\section{A CASE OF MYOSITIS OSSIFICANS.} BY WELDON CARTER, M.D. LOND., \&C.

Mrositis ossificans being a rare disease, I am induced to bring before the readers of THE LANCET the following short notes of an example which $I$ have been fortunate enough to come across, and I trust that it may prove to be an interesting addition to the report of the disease by Mr. C. Stonham which was published in THE LANCET of Dec. 31st, 1892.

A boy nine years of age first came under my observation in April, 1892, being brought to the Wigan Infirmary for certain "lumps in his back" which had been first noticed four or five years previously and had gradually increased in size and number during that period. There had never been

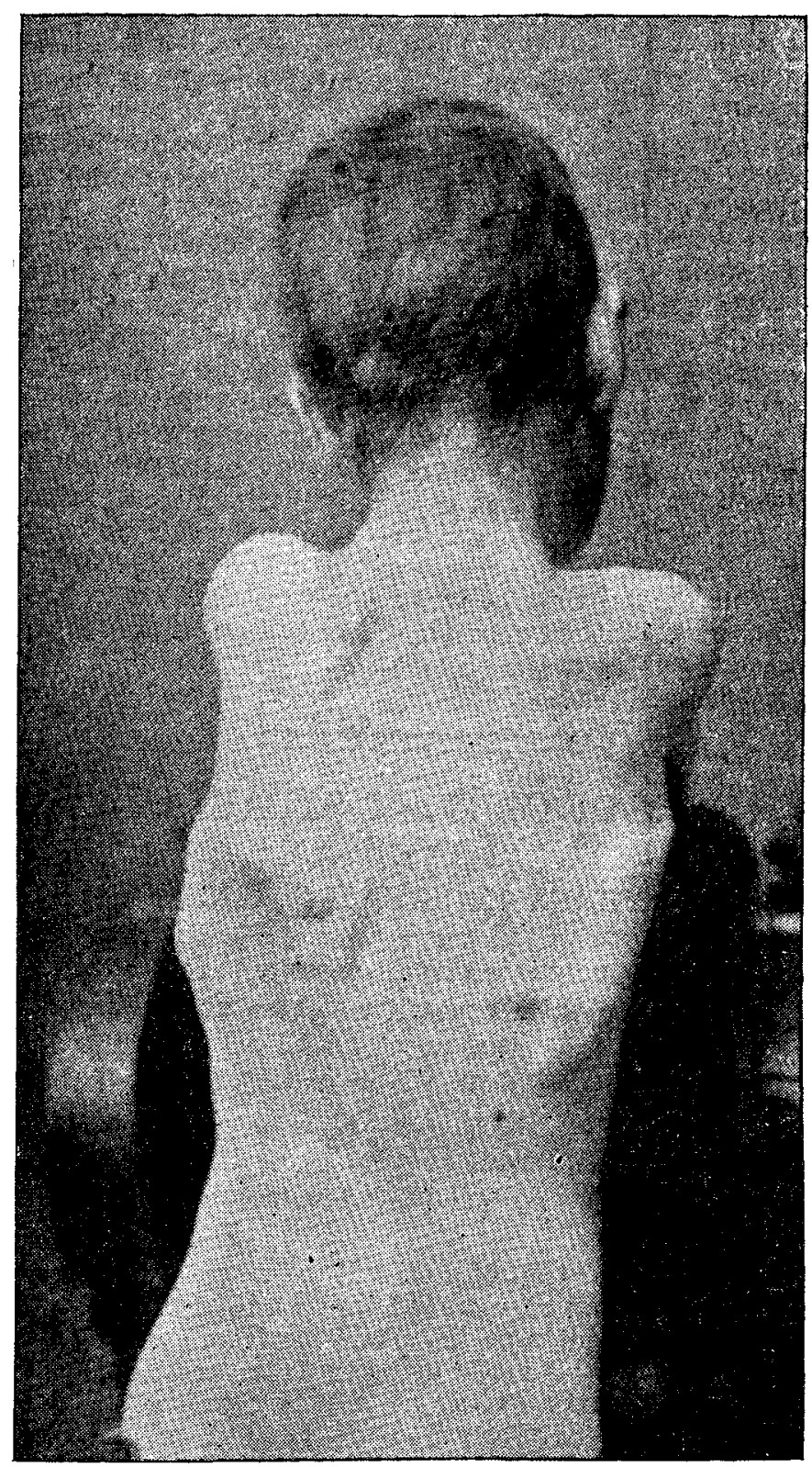

any complaint of pain or tenderness. The patient's family history was good and he had always enjoyed good health. The condition of the boy when seen by me on April 21st, 1892, was as follows. He was quite healthy in general appearance, though he stood somewhat stiffly with his head slightly bent forward, and was unable to fully extend the dorsal and lumbar portions of his spine; the cervical portion, however, presented very little alteration in mobility from the normal. On stripping the patient the lumbar portion of the erector spinæ on either side was found to be converted into a mass of bone which projected backwards and laterally more than in the case of healthy muscles; the mass measured four and a half inches transversely-i. e., two inches to the right and two and a half inches to the left of the middle line of the body. The sacral spines were rather more prominent than natural. In the posterior axillary folds there were observed irregular movable masses of considerable size which, though more prominent on the left side, occupied a greater 
length of the axillary border of the right latissimus dorsi muscle. Other movable masses were found in the teres major muscles, along the vertebral border of the right scapula, near the angle of the left scapula, and in the left trapezius muscle near its occipital attachment. The great toes were in the position of hallux valgus, the phalanges of the first, fourth, and fifth toes in both feet were very short, and there was slight webbing between the second and third toes of each foot. The hands were normal. On examining the boy on April 7th, 1893, I found that there was no increase in the stiffness of the back, but the bony masses previously noted had undergone considerable increase in size, this being especially the case with those in the latissimi dorsi. There were several fresh bosses of bony deposit to the left of the spine, commencing at about the level of the eighth dorsal vertebra and extending outwards and slightly upwards in the direction of the left scapula. Several other new nodules were discovered in different parts of the back. So far as could be made out, there was no evidence of the adductor muscles of the thighs being affected. The masses of bone were mostly of an irregularly rounded shape, but a few were somewhat spiculated. There was no indication of any old or present suppuration.

The illustration, which was taken from a photograph dated April 8th, 1893, gives a good general idea of the extent and distribution of the disease.

Remarks - This case supports the conclusions arrived at by Mr. Stonham that myositis ossificans occurs more frequently in the male sex, in youth, and without hereditary transmission; also that it commences in the muscles of the back and runs a progressive course. It is peculiar, however, in the entire absence of any history of local pains, nor is there any history of such etiological factors as cold, damp, injury, or disease, which have occasionally been met with in this affection. The case is especially interesting as showing its association with hallux valgus and microdactyle, and would add that this observation was made quite independently, for at the time of writing my original notes I was quite unaware of their occasional association, which greatly enhances the value of the observation.

Winckley-square, Preston.

\section{TWO CASES OF GLUTEAL STRAIN IN GOLF- PLAYERS.}

BY A. SYMONS ECCLES, M.B., C.M. ABERD.

THE following notes are of two cases apparently identical both in regard to their causation and nature, which came under my care in the earlier months of 1893 . Both patients described themselves as being beginners at golf and attributed the condition, from which they suffered, to the unaccustomed attitude and unskilful mode adopted by each of them in performing the act which, I believe, is technically known to golf players as "driving."

The first case was that of a man fifty-two years of age, who came into my consulting-room in February, 1893, presenting the usual appearance of a patient suffering from severe sciatica of the right limb - his mode of progression and the posture adopted in standing, being at first sight identical with those most commonly acquired by persons afflicted with long-standing painful affections of the sciatic nerve. Supporting himself on two sticks with the trunk thrown over to the left side, the right knee was semiflexed and the heel raised, the toes only being in contact with the ground. $\mathrm{He}$ complained of severe pain over the greater part of the right buttock, which was greatly increased by any movement of the right limb, especially aggravated when the weight of the trunk was thrown upon it, as when the left limb was raised in the act of walking. While standing he was careful to throw the whole weight upon the unaffected limb, and could not be persuaded to relinquish the support afforded by the sticks or to attempt locomotion without their aid. On examination I failed to detect any tenderness over the sciatic notch or anywhere in the course of the nerve, nor was there any tenderness about the buttock, save on its upper and outer aspect. Between the anterior superior spine of the ilium and the upper margin of the great trochanter, there were distinct tenderness on pressure and apparent swelling. There was also pain of a less severe character in the right lumbar region, which was increased when any attempt was made, whether passive or active, to induce the resumption of the erect posture. No tenderness, however, was elicited on pressure over the lumbar region. On measurement from the point of the second sacral vertebra round the respective buttocks to the median line in front, the swelling on the right side, which I had been at first inclined to regard as only apparent and merely due to posture, proved to be real, as on that side the measurement between the sacral spine and the middle line in front was $53 \mathrm{~cm}$., the corresponding measurement round the left buttock being only $48 \mathrm{~cm}$. The measurements were made as the patient stood supporting his weight on the left limb, the right glutei being completely relaxed, thus showing that the increased measurement was not due to the contraction of the anterior fibres of the gluteus maximus. On inquiry as to the cause and duration of the pain, the patient informed me that he had, within the previous ten days, commenced to learn the practice of golf, and though, during the first few days, going round the links once daily, he had experienced some general stiffness and soreness at night and in the morning on rising, no localised or severe pain had been felt until two days previously, when, having devoted the greater part of the afternoon to the practice of a stroke involving an attitude and a rapid muscular effort which $I$ am at a loss to describe, but which are no doubt familiar to those who play golf, and desiring to pose in driving a "tee'd ball" in the most finished style, he feit a violent pain in the upper and outer part of the buttock and over the right lumbar region, so that on leaving the ground he was unable to walk without much suffering and could not maintain the erect posture. At night the pain involved sleeplessness, and no sort of rest could be obtained save while lying on the left side with the back and right thigh propped with pillows. Hot fomentations and rubbing with embrocations bad failed to give relief.

The second case occurred in the person of a member of our own profession, who consulted my friend Mr. Keetley, to whom I am indebted for the opportunity of seeing this second example of the same accident, as he was sent to me for massage of the affected part. I first saw the patient early in April, 1893, when he told me that, as a beginner, on March 31st he was playing golf and went twice round the links, feeling very stiff at the end of the day, especially about the right hip, but not until the evening did he feel anything seriously amiss, when he began to suffer somewhat severe pain about the upper part of the right buttock and lumbar region. As time went on, he felt no better and was unable to support the weight of the body on the right limb, the trunk being tilted to the left side and the left shoulder thrown forward, the patient presenting somewhat the appearance of lateral spinal curvature, due to faulty posture. The position adopted by this patient when standing and the mode of performing the act of walking were identical with those previously described in the first case. On examination I failed to detect any tenderness on pressure; the whole buttock appeared to be slightly swollen, but the muscles were kept carefully relaxed, any attempt to throw them into action causing pain, especially between the crest of the ilium in the axillary line and the great trochanter, the pain radiating forwards towards the anterior superior spine and upwards over the right erector spinæ. The measurements from the centre of the sacrum to the median line in front over the buttock were-right $49 \mathrm{~cm}$., left $47 \mathrm{~cm}$. Firm massage of the buttock and loin was administered for fifteen minutes. On April 12th the measurements were-right $48 \mathrm{~cm}$; left $47 \mathrm{~cm}$. After two or three days' massage the severe pain subsided, though it was not until some days after this that the patient was able to assume the erect posture and to maintain it without painful effort.

In the first case daily massage of the part for seven days completely restored the patient to the painless use of the affected limb, and after the first four days he was able to walk and maintain an almost erect position, both standing and walking; but the treatment was initiated within twelve hours of the appearance of the local deformity. In the second case, however, there was a lapse of ten days between the commencement of the pain and stiffness and the date on which the patient first saw Mr. Keetley and myself. Owing to the engagements of the patient, he was unable to attend for massage regularly, otherwise I believe that the pain, swellino and deformity characteristic of this new accidental malady would have more readily been reduced. The lesion, if so it may be termed, which I venture to suggest existed in these two cases, somewhat akin in their nature and etiology to tennis-elbow, appears to me to consist of a strain and possible 